\title{
Design \& Implementation of Adaptive Fingerprint Biometrics with Training of DataSet
}

\author{
Richa $^{1}$, Ritika Saroha ${ }^{2}$ \\ Computer Science \& Engg Department, I2TB College (IGI) Jhundpur, Sonipat ${ }^{1}$ \\ Asst. Prof, Computer Science \& Engg Department, I2TB College (IGI) Jhundpur, Sonipat ${ }^{2}$
}

\begin{abstract}
This paper presents a fingerprint biometric system based on textures. In this work, it uses the training of image dataset for minimization of error. Fingerprint recognition is one of the oldest forms of biometric identification. However obtaining a good fingerprint image is not always easy. So the fingerprint image must be pre-processed before matching. The main objective of thesis is to design an image matching algorithm which is applied to any image for matching. The image will be of any size or any format. For this, the other objective is to present a better and enhanced fingerprint image. Commonly used features for improving fingerprint image quality are energy and local orientation. Accurate segmentation of fingerprint ridges from noisy background is necessary. For efficient enhancement and feature extraction algorithms, the segmented features must be void of any noise. A pre-processing method consisting of field orientation, ridge frequency estimation, filtering, segmentation and enhancement is performed. The obtained image is applied to a thinning algorithm and subsequent minutiae extraction. The methodology of image pre-processing and minutiae extraction is discussed. The simulations are performed in the MATLAB environment to evaluate the performance of the implemented algorithms. Results and observations of fingerprint images are presented at the end.
\end{abstract}

Keywords: Fingerprinting, pattern recognition, feature extraction, minutia details, analysis of fingerprints.

\section{INTRODUCTION}

Nowadays, there is an emerging interest in the application of biometric authentication and identification. Biometric identification is a growing and notorious field in which civil liberties groups express their concern over identity and privacy issues. Today, biometric laws and regulations are in process and biometric industry standard are being tested. This development has the great concern of security in the use of Internet application for consistent and automatic personal identification. Traditional automatic personal identification schemes such as knowledge based (password or personal identification number) and token based (passport, driver license, and ID card) identifications are prone to fraud because pins may be forgotten or guessed by an imposter and the tokens may be lost or stolen. Therefore, traditional knowledge-based and tokenbased approaches are unable to satisfy the security requirements of our electronically interconnected information society [17].

The selection of biometric feather depends on requirements of application. The most common biometric trait is fingerprint identification. Fingerprint recognition has been widely adopted for user identification due to its reliable performance, usability, and low cost compared with other biometric traits such as signature, iris, face, and gait recognition.

Most image-processing methods involve treating image as a two-dimensional-signal and applying standard signal processing techniques to it. Image processing is used to resolve identification problems, such as in scientific medicine or in creating weather maps from satellite pictures. Although large-scale image processing systems still are being sold for massive imaging applications, such as handling of satellite images, the tendency continues toward diminishing and blending of general -purpose small computers with specified image processing hardware [5].

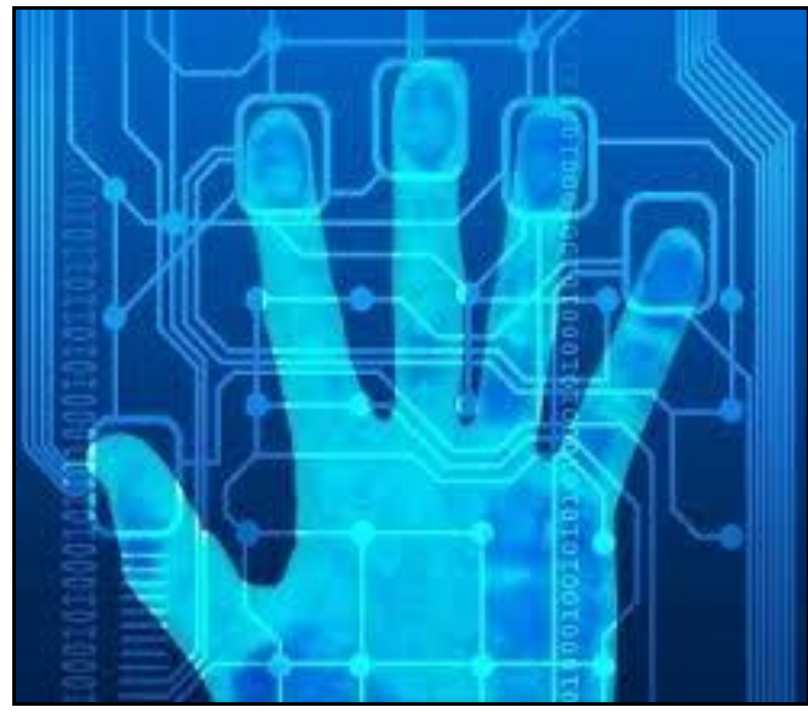

Figure 1: Fingerprint Image [1]

Skin on human fingertips contains ridges and valleys which together forms distinctive patterns. These patterns are fully established under pregnancy and are permanent throughout entire lifetime. Prints of those patterns are called fingerprints. Injuries like cuts, burns and contusions canprovisionally damage worth of fingerprints but when fully healed, patterns will be restored.Automatic fingerprint recognition has become a widely used technology in both forensicand biometrics applications. The selection of biometric feather depends on requirements of application. The most common biometric trait is fingerprint identification. 
Fingerprint recognition has been widely adopted for user identification due to its reliable performance, usability, and low cost compared with other biometric traits such as signature, iris, face, and gait recognition.

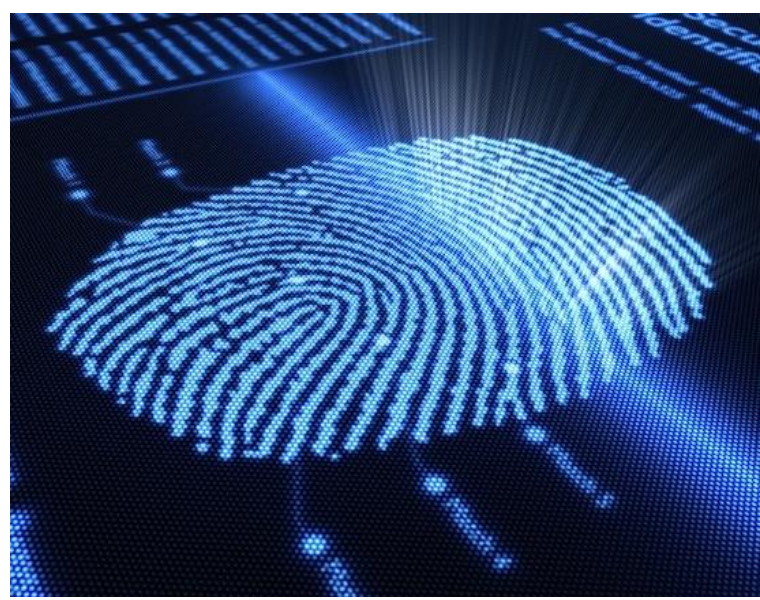

Figure 2: Fingerprint Sample Image [1]

Since biometric characteristics are distinctive, cannot be forgotten or lost, and the person to be authenticated needs to be physically present at the point of identification, biometrics is inherently more reliable and more capable than traditional knowledge-based and token-based techniques. Biometrics also has a number of disadvantages. For example, if a password or an ID card is compromised, it can be easily replaced [2].

The paper is organized as follows. In section II, we discuss related work with recognition of fingerprint images. In Section III, It describes steps of fingerprinting system. In Section IV, it describes the design and implementation algorithms used in fingerprinting image processing technique. The results are given in Section V. Finally, conclusion is explained in Section VI.

\section{RELATED WORK}

Some Authors [1] proposed an effective algorithm for fingerprint image quality improvement is proposed. The algorithm consisted of two stages. The first stage was decomposing the input fingerprint image into four subbands by applying two-dimensional discrete wavelet transform. At the second stage, the compensated image was produced by adaptively obtaining the compensation coefficient for each sub-band based on the referred Gaussian template. The method concluded an improved clarity, quality and continuity of ridge structures therefore accuracy was also increased. Background and the blurred region of fingerprint images were also removed.

Some [2] proposed a biometrics-based authentication scheme for multi-server environment using elliptic curve cryptography. To the best of our knowledge, the proposed scheme is the first truly three-factor authenticated scheme for multi-server environment. They also demonstrated the completeness of the proposed scheme using the Burrows Abadi-Needham logic.

Authors [3] presented the several improvements to an adaptive fingerprint enhancement method that was based on contextual filtering. In the global analysis and matched filtering blocks, different forms of order statistical filters were applied. These processing blocks yield an improved and new adaptive fingerprint image processing method.

Some [4] proposed a novel and effective two-stage enhancement scheme in both the spatial domain and the frequency domain by learning from the underlying images. They first enhanced the fingerprint image in the spatial domain with a spatial ridge-compensation filter by learning from the images. With the help of the first step, the second stage filter, i.e., a frequency band-pass filter that was separable in the radial- and angular-frequency domains was employed. An experimental result showed that our proposed algorithm was able to handle various input image contexts and achieves better results compared with some state-of-the-art algorithms over public databases, and to improve the performances of fingerprintauthentication systems

Some [5] proposed a high-resolution palm print recognition system based on minutiae. The proposed system followed the typical sequence of steps used in fingerprint recognition, but each step had been specifically designed and optimized to process large palm print images with a good trade-off between accuracy and speed. A sequence of robust feature extraction steps allowed to reliably detecting minutiae; moreover, the matching algorithm was very efficient and robust to skin distortion, being based on a local matching strategy and an efficient and compact representation of the minutiae. Experimental results showed that the proposed system compares very favourably with the state of the art.

Some authors [6] proposed that a novel fingerprint reconstruction algorithm is proposed to reconstruct the phase image, which is then converted into the grayscale image. The proposed reconstruction algorithm has been evaluated with respect to the success rates of type-I attack and type-II using a commercial fingerprint recognition system. The experimental results show that the reconstructed image is very consistent with the original fingerprint and that there is a high chance of deceiving a state-of-the-art commercial fingerprint recognition system. Authors [7] presented High-resolution automated fingerprint recognition systems (AFRSs) offer higher security because they were able to made use of level-3 features, such as pores, that were not available in lower resolution images. After experiments on their collected fingerprint images and applying three requirements for the proportions of minutiae and pores that must be retained in a fingerprint image, they recommended a reference resolution of $800 \mathrm{dpi}$.

Some [8] introduced a novel fingerprint matching algorithm using both ridge features and the conventional minutiae feature to increase the recognition performance against nonlinear deformation in fingerprints. Experiments were conducted for the FVC2002 and FVC2004 databases to compare the proposed method with the conventional minutiae-based method. The proposed method achieved higher matching scores as the EER \% reduced. Thus, they had concluded that the proposed ridge feature gave additional information for fingerprint matching with little increment in template size and used in conjunction with existing minutiae features to increase the accuracy and robustness of fingerprint recognition systems. 


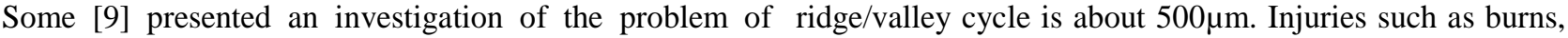
retrieving candidate lists for matching partial fingerprints abrasions, or cut do not affect the underlying ridge by exploiting global topological features. They proposed structure, and the original pattern is duplicated in any new an analytical approach for reconstructing the global skin that grows.

topology representation from a partial fingerprint. There are two main types of features in fingerprints: (i) Statistical experiments show that proposed model-based global ridge and furrow structures which form a special approach can effectively reduce the number of candidates for pair wised fingerprint matching, and thus significantly pattern in the central region of the fingerprints, and (ii) improve the system retrieval performance for partial fingerprint identification.

Authors [10] proposed the Minutia Cylinder-Code (MCC): a novel representation based on $3 \mathrm{D}$ data structures, built from minutiae distances and angles. The cylinders can be created starting from a subset of the mandatory features defined by standards like ISO/IEC 19794-2 (2005). Cylinder invariance, fixed-length, and bit-oriented coding, some simple but very effective metrics can be defined to compute local similarities and to consolidate them into a global score. MCC relied on a robust discretization of the neighbourhood of each minutia into a 3D cell-based structure named cylinder. Simple but effective techniques for the computation and consolidation of cylinder similarities were provided to determine the global similarity between two fingerprints.

\section{PROPOSED BIOMETRICS SYSTEM}

The main work of this thesis is to design an image matching algorithm which is used to compare two images using pixel based approach. This work is used for a fingerprint matching application. The work includes reading of two images firstly. One is sample image and other is victim image. After this, an enhancement algorithm is required for adjusting the contrast of images. Then this process is follows 1) Image segmentation 2) Thresholding 3) Image thinning 4) Features extraction 5) Image Matching etc. After this, the work performance is compared with matching algorithm using histogram equalization enhancement. The performance parameters like MSE, PSNR and computation time is evaluated.

A fingerprint is the print of a fingertip epidermis, produced when a finger is pressed against a smooth surface. Individual epidermal ridges and furrows have different characteristics. This forms the foundation of fingerprint. In a fingerprint image, ridges are dark whereas valleys are bright, as show in Fig.3.

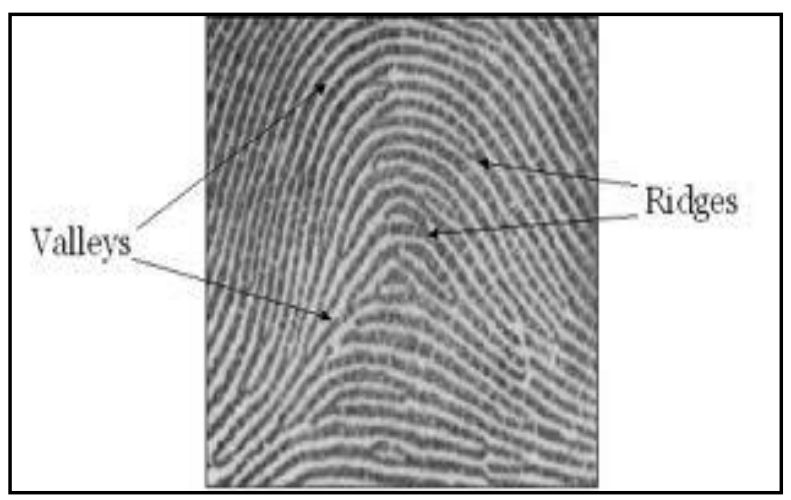

Figure 3: A Fingerprint image showing ridges and valley [8]

Ridge varies in width from $100 \mu \mathrm{m}$, for very thin ridges, to $300 \mu \mathrm{m}$ for thick ridges. Generally the period of a minute details associated with local ridges and furrows. A fingerprint is typically classified based on only the first type of features and uniquely identified based on the second type of features. The minutiae-based representation is the most popular representation of fingerprints as it has a long history of use by the forensic experts who visually match fingerprints.

A fingerprint recognition system constitutes of fingerprint device, minutia extractor and minutia matching. For fingerprint acquisition, optical or semi-conduct sensors are extensively used. They have large efficiency and acceptable accuracy except for some cases that user's finger is too dirty or dry.
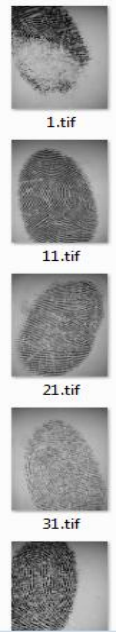
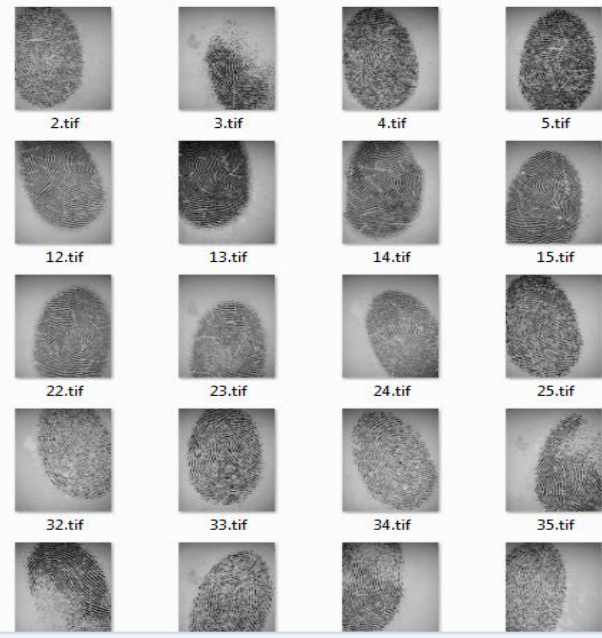

Figure 4: Fingerprint Database Images
Image acquisition is first step in the approach. It is very important as quality of the fingerprint image must be good and free from any noise. A good fingerprint image is desirable for better performance of fingerprint algorithms. Based on the mode of acquisition, a fingerprint image may be divided into off-line or live-scan. An off-line image is obtained by smearing ink on fingertip and creating an inked impression of fingertip on paper.

Fingerprint image quality is an important factor in performance of minutiae extraction and matching algorithms. A good quality fingerprint image has high contrast between ridges and valleys. A poor quality fingerprint image is low in contrast, noisy, wrecked, or smudgy, causing spurious and missing minutiae. Poor quality can be due to cuts, creases, or bruises on surface of fingertip, excessively wet or dry skin condition, uncooperative attitude of subjects, broken and unclean scanner devices, low quality fingers (elderly people, manual worker), and other factors.

Segmentation of an image entails the division or separation of the image into regions of similar attribute. The most basic attribute for segmentation is image 
luminance amplitude for a monochrome image and color components for a color image. Image edges and texture are also useful attributes for segmentation. In general, only a Region of Interest (ROI) is useful to be recognized for each fingerprint image.

Fingerprints have local parallel ridges and valleys, and well-defined local frequency and orientation. Properly tuned Gabor filters can remove noise, preserve the true ridge and valley structures, and provide information contained in a particular orientation in the image. A minutia point can be viewed as an anomaly in locally parallel ridges and it is this information that we are attempting to capture using the median filters.

The category of a fingerprint is determined by its global ridge and furrow structures. A valid feature set for fingerprint classification should be able to capture this global information effectively. After enhancement of the fingerprint image, image is ready for minutiae extraction. For proper extraction, however, a thinning algorithm is applied to enhanced image. It produces a skeletonised representation of image.

Thinning is a morphological operation that is used to remove selected foreground pixels from binary images. It is used to eliminate redundant pixels of ridges till ridges are just one pixel wide. Thinning is normally only applied to binary images, and creates another binary image as output. It is final step prior to minutiae extraction.

The requirements of a decent thinning algorithm are

a) The thinned fingerprint image attained should be of single pixel width.

b) Each ridge must be thinned to its middle pixel.

c) Noise and particular pixels must be eliminated.

After the enhancement of fingerprint image the next step is minutiae extraction. The method extracts minutiae from the enhanced image. This method extracts the ridge endings and bifurcations from skeleton image by examining the local neighbourhood of each ridge pixel using a $3 \times 3$ window. The pre-processing stage does not totally heal the fingerprint image. For instance, false ridge breaks due to insufficient amount of ink and ridge cross-connections due to over inking are not totally eliminated. Actually all earlier stages themselves occasionally introduce some artifacts which later lead to spurious minutia. This false minutia will expressively affect the accuracy of matching if they are simply regarded as genuine minutia.

Given two set of minutia of two fingerprint images, minutia match algorithm determines whether the two minutia sets are from the same finger or not. An alignment-based match procedure is used. It includes two consecutive stages: one is alignment stage and second is match stage.

A. Proposed Parameter: Signal Quality

This term is often used to characterise the signal at the output of decoder. There is no universally accepted measure for signal quality. One measure that is often cited is the signal to noise ratio $S N R$, which can be expressed as eq. (1):

$$
S N R=10 \log 10 \frac{\text { Input signal energy }}{\text { noise error }}
$$

The noise signal energy is defined as energy measured for a hypothetical signal is difference between the encoder input signal and the decoder output signal. Note that, SNR defined is given in decibels $(\mathrm{dB})$. In the case of images, $P S N R$ (peak signal-to-noise ratio) is used instead of $S N R$

\section{RESULTS}

Fingerprint matching is the process used to determine whether two sets of fingerprint Ridge detail comes from the same finger. There exist multiple algorithms that do fingerprint matching in many different ways. Some methods involve matching minutiae points between the two images, while others look for similarities in the bigger structure of the fingerprint. For fingerprint matching, it requires two input images. One is of victim and one for comparison.

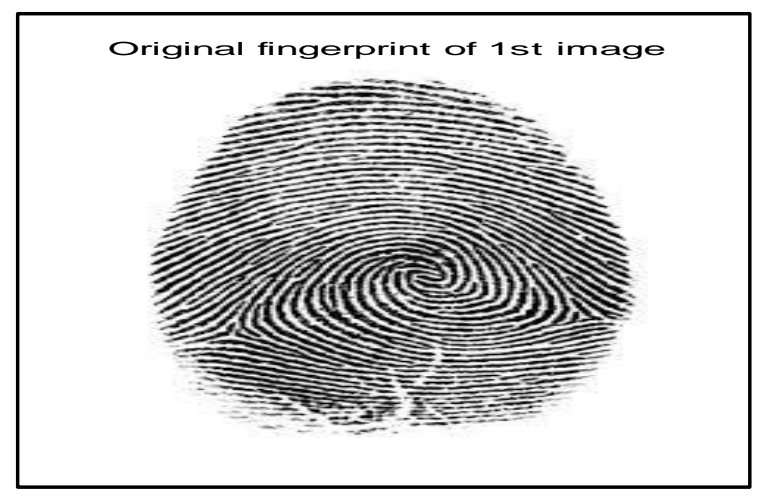

Figure 5: Original First Fingerprint Sample

Original fingerprint of 2 nd image

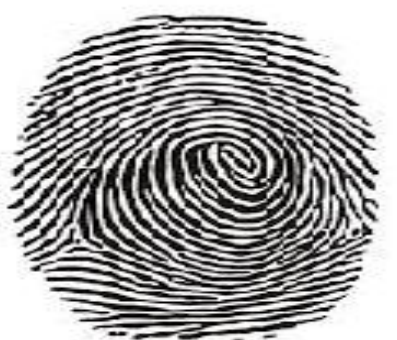

Figure 6: Original Second Fingerprint Sample

\begin{tabular}{|c|c|c|c|c|}
\hline Iteration & $: 26493$ & mse & $=$ & 0.000100 \\
\hline Iteration & $: 26494$ & mse & $:$ & 0.000100 \\
\hline Iteration & $: 26495$ & mse & : & 0.000100 \\
\hline Iteration & $: 26496$ & mse & : & 0.000100 \\
\hline Iteration & $=26497$ & mse & : & 0.000100 \\
\hline Iteration & $: 26498$ & mse & : & 0.000100 \\
\hline Iteration & $: 26499$ & mse & : & 0.000100 \\
\hline Iteration & $: 26500$ & mse & : & 0.000100 \\
\hline Iteration & $: 26501$ & mse & : & 0.000100 \\
\hline Iteration & $: 26502$ & mse & : & 0.000100 \\
\hline Iteration & $: 26503$ & mse & $:$ & 0.000100 \\
\hline Iteration & $: 26504$ & $\begin{array}{l}\text { mse } \\
\text { mse }\end{array}$ & 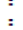 & 0.000100 \\
\hline Iteration & $: 26505$ & mse & : & 0.000100 \\
\hline Iteration & $: 26506$ & mse & : & 0.000100 \\
\hline Iteration & $: 26507$ & mse & : & 0.000100 \\
\hline Iteration & $: 26508$ & mse & $:$ & 0.000100 \\
\hline Iteration & $: 26509$ & mse & $:$ & 0.000100 \\
\hline Iteration & $: 26510$ & mse & : & 0.000100 \\
\hline Iteration & $: 26511$ & mse & : & 0.000100 \\
\hline Iteration & $: 26512$ & mse & $:$ & 0.000100 \\
\hline Iteration & $: 26513$ & mse & : & 0.000100 \\
\hline Iteration & $: 26514$ & mse & : & 0.000100 \\
\hline Iteration & $: 26515$ & mse & : & 0.000100 \\
\hline Iteration & $: 26516$ & mse & $:$ & 0.000100 \\
\hline Iteration & $: 26517$ & mse & $:$ & 0.000100 \\
\hline Iteration & $: 26518$ & mse & $=$ & 0.000100 \\
\hline Iteration & $: 26519$ & mse & $:$ & 0.000100 \\
\hline Iteration & $: 26520$ & mse & $=$ & 0.000100 \\
\hline
\end{tabular}


INTERNATIONAL JOURNAL OF INNOVATIVE RESEARCH IN ELECTRICAL, ELECTRONICS, INSTRUMENTATION AND CONTROL ENGINEERING Vol. 3, Issue 12, December 2015

Figure 7: Training Output of Image Dataset

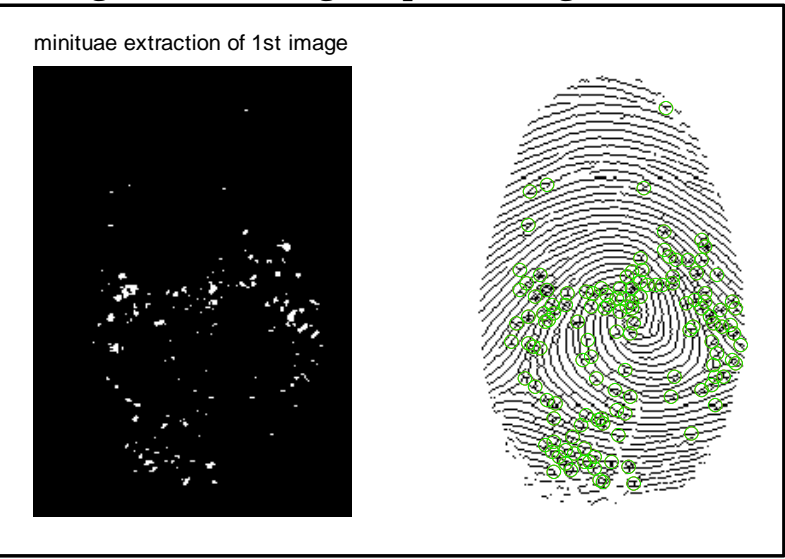

Figure 8: Minutia Extraction of Input Image

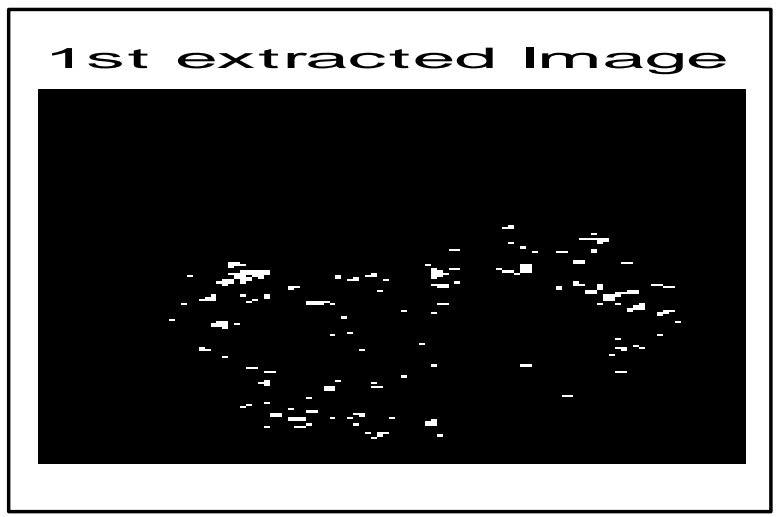

Figure 9: Feature Extraction of $1^{\text {st }}$ Image

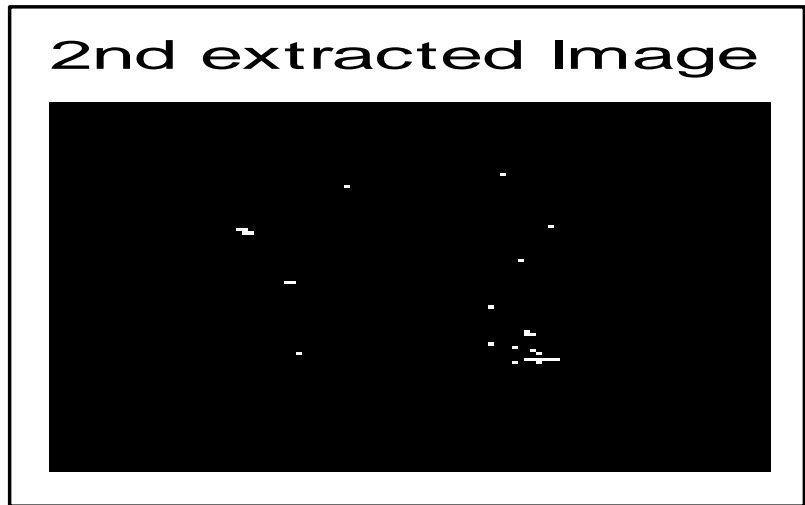

Figure 10: Feature Extraction of $2^{\text {nd }}$ Image

\section{difference detected}

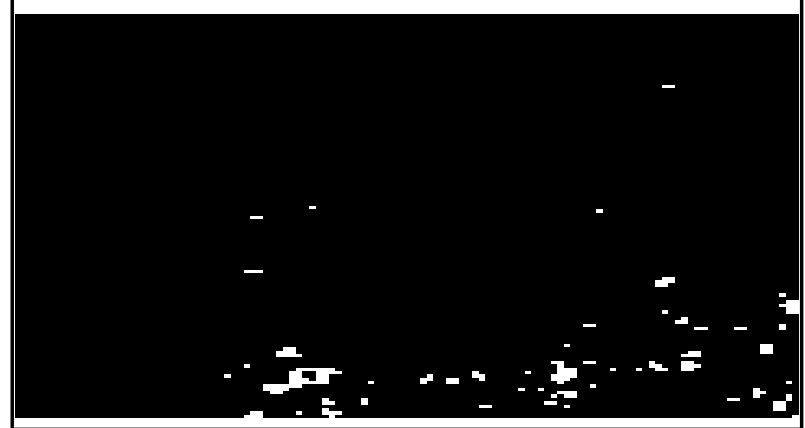

Figure 11: Image Matching Output

\section{CONCLUSION}

The goal of this work is to design a system of image matching. It is used in the application of fingerprint matching system. It is used to study the security impact of partial fingerprints on automatic fingerprint recognition systems and to develop an automatic system that can overcome the challenges presented by partial fingerprint matching.The proposed algorithm is implemented in MATLAB. In this work, it uses the training of image dataset for minimization of error. The number of processes is used to match these images. This algorithm is able to get good contrasted image which increases the brightness of the low contrasted images. This algorithm is tested on different type of images. The experimental results show that the brightness is increased as compared to previous one. The proposed system shows that it provides minimum error value by training of image dataset.

\section{REFFERENCES}

[1] Jing-Wein Wang, Ngoc Tuyen Le, Chou-Chen Wang, and JiannShu Lee, " Enhanced Ridge Structure for Improving Fingerprint Image Quality Based on a Wavelet Domain”, 2015 IEEE

[2] Debiao He, and Ding Wang, "Robust Biometrics-Based Authentication", 2014 IEEE Scheme for Multiserver Environment.

[3] Josef Strom Bart unek, Mikael Nilsson, Benny Sallberg, and Ingvar Claesson,, "Adaptive Fingerprint Image Enhancement With Emphasis on Preprocessing of Data", 2013 IEEE

[4] Jucheng Yang, Naixue Xiong, and Athanasios V. Vasilakos, "TwoStage Enhancement Scheme for Low-Quality Fingerprint Images by Learning From the Images" 2013 IEEE

[5] Raffaele Cappelli, Matteo Ferrara, and Dario Maio, "A Fast and Accurate Palmprint Recognition System Based on Minutiae” 2012 IEEE

[6] Jianjiang Feng,Member, and Anil K. Jain, "Fingerprint Reconstruction: From Minutiae to Phase” 2011 IEEE

[7] David Zhang, Feng Liu, Qijun Zhao, Guangming Lu, and Nan Luo, "Selecting a Reference High Resolution for Fingerprint Recognition Using Minutiae and Pores" 2011 IEEE

[8] Heeseung Choi, Kyoungtaek Choi, and Jaihie Kim, "Fingerprint Matching Incorporating Ridge Features With Minutiae" 2011 IEEE

[9] Yi (Alice) Wang, Jiankun Hu, "Global Ridge Orientation Modeling for Partial Fingerprint Identification” 2011 IEEE

[10] Raffaele Cappelli, Matteo Ferrara, Davide Maltoni, "Minutia Cylinder-Code: A New Representation and Matching Technique for Fingerprint Recognition" 2010 IEEE

[11] Praveer Mansukhani, Sergey Tulyakov, and Venu Govindaraju, “A Framework for Efficient Fingerprint Identification Using a Minutiae Tree" 2010 IEEE

[12] Lavanya B N, K B Raja, Venugopal K R and L M Patnaik, "Minutiae Extraction in Fingerprint using Gabor Filter Enhancement" 2009 IEEE

[13] Fanglin Chen, Jie Zhou and Chunyu Yang, "Reconstructing Orientation Field From Fingerprint Minutiae to Improve MinutiaeMatching Accuracy" 2009 IEEE

[14] Weiguo Sheng, Gareth Howells, Michael Fairhurst, and Farzin Deravi, “A Memetic Fingerprint Matching Algorithm” 2007 IEEE

[15] Arun Ross, Jidnya Shah, and Anil K. Jain, "From Template to Image: Reconstructing Fingerprints from Minutiae Points" 2007 IEEE

[16] Xudong Jiang, Manhua Liu, and Alex C. Kot, "Fingerprint Retrieval for Identification" 2006 IEEE

[17] R. Gonzalez, R. Woods and S. Eddins "Digital Image Processing Using Matlab”, 2004, Prentice Hall. 